\title{
Effects of cellulose adulteration on maintenance-feeding behavior in rats with VMH lesions
}

\author{
PAUL J. WELLMAN and RONALD H. PETERS \\ Iowa State University, Ames, Iowa 50010
}

\begin{abstract}
Female rats with lesions of the ventromedial hypothalamus (VMH) overate and gained more body weight than same-diet control rats, following extended maintenance on a ground-chow diet adulterated with an equal weight of cellulose. Adulteration with cellulose clearly decreased diet palatability, because control rats fed the cellulose-adulterated diet ate and weighed less than control rats fed the unadulterated ground-chow diet. The overeating of a less palatable diet by VMH rats is consistent with the hypothesis that VMH lesions increase hunger motivation.
\end{abstract}

Lesions of the ventromedial hypothalamus (VMH) in rats induce hyperphagia and rapid weight gain (dynamic stage), followed by near-normal food intake as body weight becomes asymptotic at an elevated plateau (static stage). One striking aspect of the feeding behavior of obese VMH rats is their extraordinary sensitivity to manipulations of diet palatability. They clearly undereat and lose more weight than control rats when their diets are made less palatable (Graff \& Stellar, 1962; Powley, 1977; Teitelbaum, 1955). It is likely, however, that these exaggerated reactions to palatability manipulations are a consequence of some correlate of obesity, rather than VMH damage per se, since VMH rats fed diets described as unpalatable maintain their body weights at control levels when at or near control body weights (Ferguson \& Keesey, 1975; Franklin \& Herberg, 1974). Furthermore, neurologically intact rats made obese by dietary manipulations react as do obese VMH rats when diet palatability is manipulated (Maller, 1964; Sclafani \& Springer, 1976).

Peters, Luttmers, Gunion, and Wellman (1978) recently demonstrated that VMH rats differentially consumed two quinine-adulterated maintenance diets. VMH rats fed a quinine-adulterated wet-mash diet underate and subsequently maintained their body weights below the level of same-diet control rats. In contrast, VMH rats fed a quinine-adulterated high-fat diet overate and subsequently maintained their body weights above the levels of same-diet control rats. The latter observation represents the first and only demonstration to date that $\mathrm{VMH}$ rats will overeat a maintenance diet adulterated with quinine in a concentration that induced undereating and weight loss in control rats. Since this outcome

This report is based on a thesis submitted by Paul J. Wellman to Iowa State University. Portions of this paper were presented at the Midwestern Psychological Association, Chicago, 1978. Requests for reprints should be sent to Ronald H. Peters. may be peculiar to this particular dietary adulteration, nonnutritive cellulose was used in the present experiment to adulterate a ground-chow maintenance diet for $\mathrm{VMH}$ and control rats.

\section{METHOD}

\section{Animals}

The animals were 42 female Long-Evans hooded rats (Blue Spruce Farms, Inc.), 80-90 days old and weighing 190-240 g at the beginning of the experiment. The rats were housed singly in standard wire-mesh cages in a temperature-controlled $\left(24^{\circ} \mathrm{C}\right)$ room illuminated from 0800 to $2000 \mathrm{~h}$. Tap water was available throughout the experiment, except as noted below.

\section{Diets}

Two diets were prepared and placed in wide-mouth glass jars ( $85 \mathrm{~mm}$ high with a $71-\mathrm{mm}$ opening) wired to the cage front. The ground-chow diet consisted simply of ground laboratory chow (Purina), while the adulterated form of the ground-chow diet (cellulose-chow) consisted of equal parts by weight of groundchow and nonnutritive cellulose (Teklad, Inc.).

\section{Surgery and Histology}

Food and water were removed for $24 \mathrm{~h}$ before and after surgery. All rats were anesthetized (Myothesia, $42 \mathrm{mg} / \mathrm{kg} \mathrm{IP)}$ and given methyl atropine (10 mg/kg IP) to reduce respiratory complications. Two groups $(\mathrm{N}=13$ each) received bilateral lesions with the tip of the electrode positioned $5.8 \mathrm{~mm}$ anterior to the interaural line, $.7 \mathrm{~mm}$ lateral from the midline, and $.5 \mathrm{~mm}$ above the base of the brain. The upper incisor bar was $5.0 \mathrm{~mm}$ above the interaural line. Lesions were made by passing $2.0-\mathrm{mA}$ anodal current for $20 \mathrm{sec}$ between a 30-ga nichrome steel electrode, insulated except at its conical tip, and a rectal cathode. Two groups $(\mathrm{N}=8 \mathrm{each})$ served as sham-operated controls. These rats received scalp incisions, but skull holes were not drilled.

Lesioned rats were sacrificed at the end of the experiment with chloroform and perfused intracardially with saline, followed by $10 \%$ Formalin. After fixation for a minimum of $48 \mathrm{~h}$ in $10 \%$ Formalin. $100-\mu$ sections through the VMH in the coronal plane described by König and Klippel (1963) were photographically enlarged to assess the extent and location of tissue damage.

\section{Procedure}

All rats were fed the ground-chow diet for 9 days prior to surgery. During Days 1-46 following surgery, one VMH group (VU) and one control group (CU) continued maintenance on the 
ground-chow diet while the remaining VMH and control groups (VC and CC) began maintenance on the cellulose-chow diet. Because Corbit and Stellar (1964) reported that static VMH rats previously fed a ground-chow diet exhibited additional hyperphagia and an elevation in their static body weight when shifted to a pellet diet, all rats were shifted to laboratory pellets (Teklad, Inc.) during Days 47-55. In addition, VMH and control rats previously fed the cellulose-chow diet continued maintenance on the pellet diet during Days 55-70 to identify hyperphagic VMH rats. Throughout the experiment, body weights and :4-h food intakes (corrected for spillage) were recorded every 3 rd day.

\section{RESULTS}

Data were analyzed only for those lesioned rats (VS, $\mathrm{N}=5$; VU, $\mathrm{N}=9$ ) with both bilateral VMH destruction and a weight gain following access to the pellet diet that exceeded the largest weight gain of a control rat. Such lesions were generally large, with bilateral damage including much of the area medial to the plane of the fornix, ventral to the dorsomedial nuclei, rostral to the premammillary nuclei, and caudal to the anterior hypothalamus. In contrast, the lesions that did not induce large weight gains were typically more posterior and/or asymmetrical with respect to the midline, thereby sparing much of the VMH.

Surgical and dietary manipulations induced changes in feeding behavior (Figures 1 and 2) and corresponding changes in body weight (Figure 3). Figures 1 and 2 depict weight of ground-chow intake and total weight of diet intake, respectively. Celluloseadulteration doubled the weight and approximately trebled the volume required for equivalent caloric intake. Although control rats fed the cellulose-chow diet nearly doubled the total weight of their intake (Figure 2), their caloric intake (Figure 1) was significantly lower (overall means, $t=2.56$, df $=14$, $\mathrm{p}<.05)$ than that of control rats fed the groundchow diet. As a consequence, body-weight curves for the two control groups gradually diverged during the experiment (18-g difference between group means on Day 46 was significant, $\mathrm{t}=2.48$, df $=14, \mathrm{p}<.05$ ). Body-weight differences between the two control groups near the end of this portion of the experiment were maintained, even though ground-chow intakes for the two groups were nearly identical.

VMH rats overate the ground-chow diet and displayed weight gains comparable to those typically seen when such rats are fed pellet diets. As body weight approached an elevated asymptote, groundchow intake gradually declined to near-normal levels. Although both the presence (Teitelbaum, 1955) and absence (Corbit \& Stellar, 1964; Panksepp, 1971) of hyperphagia have been reported for VMH rats fed ground-chow diets, the present data again demonstrate that VMH rats are hyperphagic and gain considerable body weight when fed ground-chow diets. Further, when VMH and control rats fed the ground-

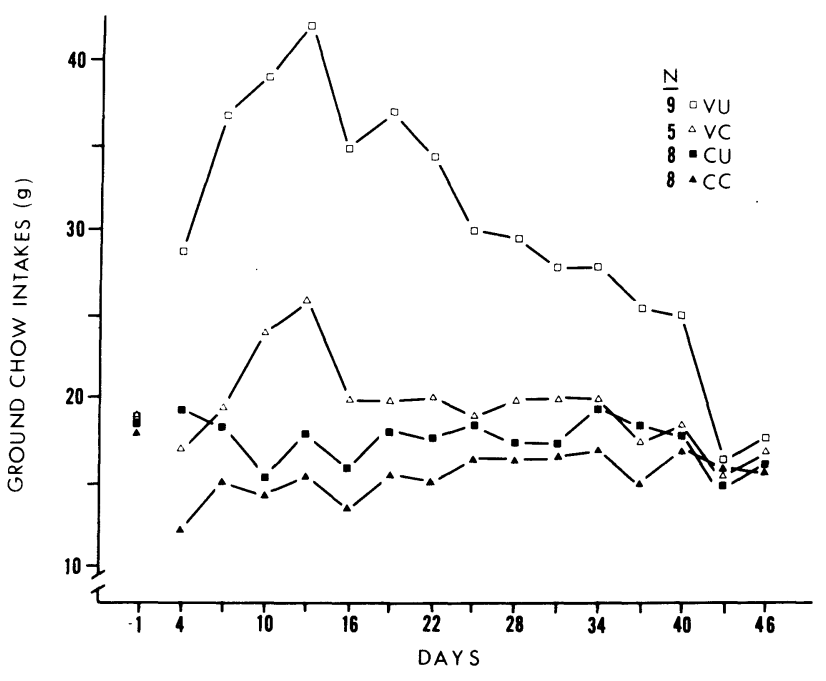

Figure 1. Mean group 24-h intakes (ground-chow weight) on the day before surgery to Day $\mathbf{4 6}$ following surgery. Lesion and diet conditions are designated by combination of the letters: C(control), V (VMH), U (unadulterated), and C (Cellulose).

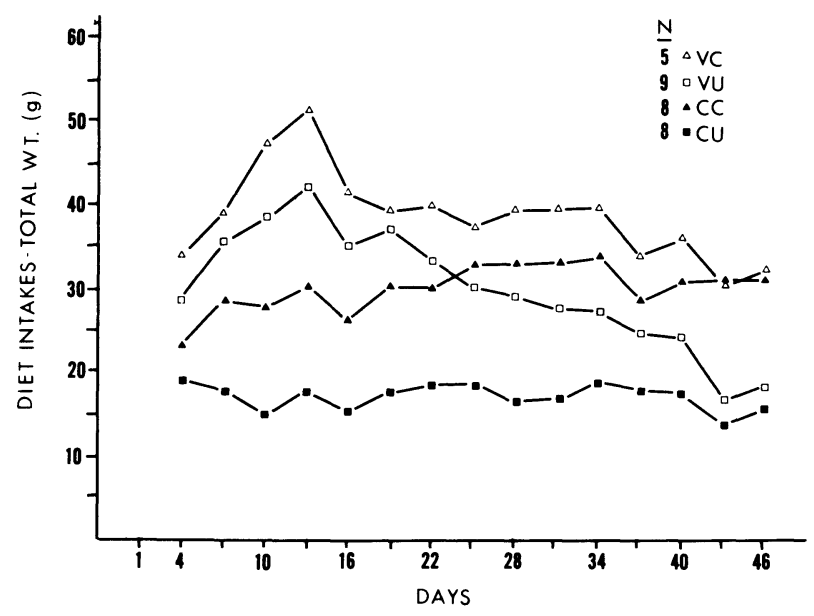

Figure 2. Mean group 24-h intakes (total diet weight) on the day before surgery to Day $\mathbf{4 6}$ following surgery.

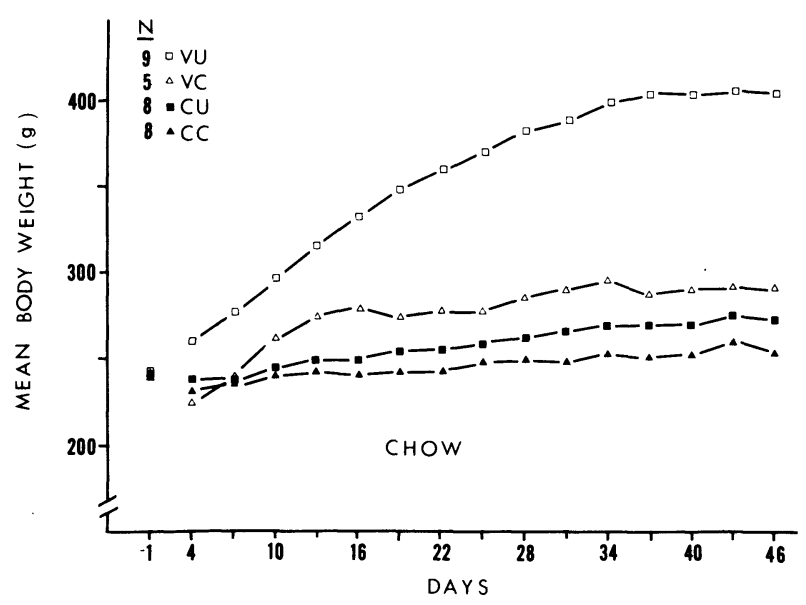

Figure 3. Mean group body weights on the day before surgery to Day 46 following surgery. 
chow diet were shifted to the pellet diet during Days 47-55, VMH rats gained less additional weight $(.5 \mathrm{~g} /$ day $)$ than control rats $(1.0 \mathrm{~g} /$ day $)$. Texture differences between ground-chow and pellet diets do not always have a major influence on the feeding behavior of $\mathrm{VMH}$ rats.

Although VMH rats fed the cellulose-chow diet initially weighed less than either control group, they subsequently more than compensated for the caloric dilution of their diet, and, by Day 10, weighed significantly more than either control group $(t=2.54$ and 2.61 , df $=11, \mathrm{p}<.05)$. VMH rats fed the cellulose-chow diet consumed significantly more ground chow than same-diet control rats (overall means, $\mathrm{t}=4.96$, $\mathrm{df}=11, \mathrm{p}<.01$ ), but less than VMH rats fed the ground-chow diet.

The mean body weight ( $294 \mathrm{~g}$, Day 46) of VMH rats fed the cellulose-chow diet significantly $(\mathrm{t}=$ 3.1, $\mathrm{df}=11, \mathrm{p}<.01$ ) exceeded that attained by control rats $(253 \mathrm{~g})$ fed the same diet. Body-weight differences were maintained, even as intakes for the two groups converged to the same level. Although the term static is typically used to describe the conjoint attributes of obesity and near-normal caloric intake when VMH rats are fed diets that induce considerable weight gain, this pattern was apparent in all three between-group comparisons described above, including the comparison between control groups fed different diets. Body-weight differences were maintained, even when caloric intakes were substantially identical. The magnitudes of body-weight differences were simply smaller when VMH and control rats were fed cellulose-adulterated diets, or when control rats were fed either ground-chow or cellulose-chow diets.

Finally, when Groups VC and CC were shifted to the pellet diet (Days 47-70), the mean body weight of VMH rats rapidly approached a higher asymptotic level (392 g, Day 70) than that attained by control rats $(280 \mathrm{~g})$.

\section{DISCUSSION}

Cellulose has frequently been employed as a dietary adulterant (Maller, 1964; Teitelbaum, 1955). In the present experiment, neurologically intact rats fed a cellulose-adulterated diet increased their total diet consumption almost twofold; however, these rats failed to consume enough of the adulterated diet to maintain caloric balance, and therefore gradually lost body weight during the course of the experiment. The failure of intact rats to maintain caloric balance cannot be due simply to the inability of their digestive system to accommodate the sheer bulk of the cellulose diet, since VMH rats consumed even more of this diet than did control rats. Cellulose adulteration appears to represent a manipulation of diet palat- ability; cellulose-adulterated diets are likely less palatable than unadulterated diets, for neurologically intact rats.

In the present experiment, $\mathrm{VMH}$ rats ate and weighed less when their ground-chow diet was adulterated with cellulose, but, more importantly, ate and weighed more than control rats fed the same diet. This observation contrasts with the report of Levison, Frommer, and Vance (1973) in which VMH rats underate with respect to same-diet control rats as the dilution of their diets with nonnutritive kaolin increased above $20 \%$. Their lesioned rats, however, weighed an average of approximately $80 \mathrm{~g}$ more than control rats at the beginning of testing, and each adulterated diet was presented for only 3 consecutive days, followed by at least 4 days' access to unadulterated chow between test diets. Their data probably would have more closely approximated ours had they used less obese rats and longer maintenance test periods. VMH rats fed the cellulose-chow diet, in this study, weighed less than either control group on Day 4, and were likely undereating the diet prior to that time.

Peters et al. (1978) demonstrated that VMH rats overate a quinine-adulterated high-fat diet, with respect to same-diet control rats. The present data extend the generality of that observation, by demonstrating that the overeating of a diet that has been made less palatable is not unique to adulteration of a high-fat diet with quinine. VMH rats also overeat a ground-chow diet adulterated with cellulose, in a concentration that induced weight loss in control rats.

In congruence with our previous interpretation (Peters et al., 1978), these data suggest that deficits in feeding behavior that are a consequence of VMH destruction are not a reflection of the animal's hyperreactivity to diet palatability, but rather occur because this destruction has, in some fashion (e.g., Friedman $\&$ Stricker, 1976), increased the animal's hunger. The animal responds appropriately by increasing its consumption of the diet made available. The body weights eventually maintained by such animals are a function of the palatability of the diets they are fed, as are the body weights of intact animals.

\section{REFERENCES}

Corbit, J. D., \& STellar, E. Palatability, food intake and obesity in normal and hyperphagic rats. Journal of Comparative and Physiological Psychology, 1964, 58, 63-67.

Ferguson, N. B. L., \& KeEsey, R. E. Effect of a quinineadulterated diet upon body weight maintenance in male rats with ventromedial hypothalamic lesions. Journal of Comparative and Physiological Psychology, 1975, 89, 478-488.

FrankiIN, K. B. J., \& Herberg, L. J. Ventromedial syndrome: The rat's "finickiness" results from the obesity, not from the lesions. Journal of Comparative and Physiological Psychology, 1974, 87, 410-414. 
Friedman, M. I., \& STricker, E. The physiological psychology of hunger: A physiological perspective. Psychological Review, 1976, 83, 409-431.

Graff, H., \& Stellar, E. Hyperphagia, obesity and finickiness. Journal of Comparative and Physiological Psychology, 1962, 55, 418-424.

König, J. F. R., \& KLIPPEL, R. A. The rat brain. Baltimore: Williams and Wilkins, 1963.

Levison, M., Frommer, G., \& Vance, W. B. Palatability and caloric density as determinants of food intake in hyperphagic and normal rats. Physiology \& Behavior, 1973, 10, 455-462.

MALLER, $O$. The effect of hypothalamic and dietary obesity on taste preference in rats. Life Sciences, 1964, 3, 1281-1291.

PANKSEPP, J. A re-examination of the role of the ventromedial hypothalamus in feeding behavior. Physiology \& Behavior, 1971, 7, 385-394.
Peters, R. H., Luttmers, L. L., Gunion, M. W., \& Wellman, P. J. Ventromedial hypothalamic syndrome: Finickiness? Physiology \& Behavior, 1978, 20, 279-285.

PowLEY, T. L. The ventromedial hypothalamic syndrome, satiety, and a cephalic phase hypothesis. Psychological Review, 1977, 84, 89-126.

SClafani, A., \& SPRINGER, D. Dietary obesity in adult rats: Similarities to hypothalamic and human obesity. Physiology \& Behavior, 1976, 17, 461-471.

Teitelbaum, P. Sensory control of hypothalamic hyperphagia. Journal of Comparative and Physiological Psychology, 1955, 48, 156-163.

(Received for publication May 25, 1978; revision accepted August 15, 1978.) 